\title{
INNOVATIVE SENSING SOLUTION AND SHM FOR MOEL-Y-PARC TELECOMMUNICATION HIGH GUYED MAST
}

\author{
K.Y. Koo ${ }^{1 *}$, J.A. Jiménez Capilla ${ }^{2}$ and J.M.W. Brownjohn ${ }^{1}$ \\ ${ }^{1}$ University of Exeter, Exeter, UK \\ ${ }^{2}$ Arqiva, Winchester, $U K$ \\ * Corresponding author
}

\begin{abstract}
This study presents the innovative sensing solution for structural health monitoring on the Moel-y-Parc telecommunication highguyed mast (HGM), together with the data interpretations from the measured accelerations and wind data. HGMs, transmitting radio and television broadcasts over the UK, are a special type of structure, extremely slender and flexible with low damping. Changing broadcast requirements in the UK have led to the need to replace or augment antennas on HGMs, but limited understanding on the dynamic behaviours of HGMs and old design codes have compromised the ability to assess safety and serviceability. Arqiva, a telecommunication company providing broadcasting facilities, owns the Moel-y-Parc HGM and has recently installed a pair of tuned liquid dampers to control potential vortex-induced oscillations due to changes in the antenna, and a structural health monitoring system has been installed to monitor effectiveness of the dampers. An innovative sensing solution was developed to monitor wind and response comprising five acceleration measurement boxes with two single-axis accelerometers each installed along the height of the mast, and one ultrasonic anemometer box installed around the top of the mast. A fibre-optic Ethernet network was constructed for communication between the sensor boxes and the base server at the ground to avoid problems related to the RF interference on signal cables by the high-density RF waves around the mast. The sensing solution performs analogue-to-digital conversions at each sensor box with time-synchronisation between the boxes managed by the resampling technique on accurately time-stamped measurements, where the timestamps are provided by a low-cost GPS module and Arduino in each box.
\end{abstract}

\section{Introduction}

A large number of high guyed masts (HGMs) were constructed In the UK between 1960 and 1980 for the BBC and the Independent Broadcasting Authority (IBA) for long range analogue television and radio broadcast and ultimately ended up being operated by Arqiva. Arqiva takes the similar role to telecoms infrastructure for broadcasters as Network Rail takes for train operating companies and has similar responsibilities and penalties for infringing service level agreements (SLAs) on customer signal reception. Up to $350 \mathrm{~m}$ tall (in the UK), HGMs have varied typologies, the majority being triangular lattice frames with solid tubular verticals, while five use $\boldsymbol{\phi} 2.7 \mathrm{~m}$ or $2.3 \mathrm{~m}$ cylindrical sections. In parallel with many other infrastructure types HGMs age beyond their original design life and Arqiva faces the challenge of assuring their serviceability and safety as they are required to accommodate new types of antennae, including heavier 5G devices. Other operators in the UK (e.g. Ministry of Defence) and worldwide face similar challenges much of the focus among members of the International Association of Shell and Spatial Structures (IASS) (WG4 IASS, 1981) is managing serviceability due to in-wind dynamic performance, considering issues such as aerodynamics and effects of new antenna, guy galloping and in particular vortex-shedding.

Catastrophic structural failure of HGMs is seldom due to wind and fatigue is uncommon but the infamous collapse of the 386m Emley Moor HGM in 1969 illustrates an unresolved issue. Collapse was due to galloping of iced stays coincident with vortex shedding on the $\boldsymbol{\phi} 2.7 \mathrm{~m}$ cylindrical tower, and excessive antenna movement due to vortex shedding remains the dominant problem for HGM dynamics due to SLAs with broadcasters which require coverage of $99.99 \%$ of population in a target area all the time or vice versa. Because broadcast signals are highly focussed in a barrow horizontal beam, rotational components of HGM vibration due to vortex shedding around antennae lead to loss of serviceability and need to be controlled.

This is the present problem caused by clearance of the 700 $\mathrm{MHz}$ (Freeview) band for mobile services, which requires installation of new $800 \mathrm{MHz}$ band antennae while the $700 \mathrm{MHz}$ antenna are still in use. These wrap-around antennae compromising multiple square panels are effectively large diameter cylinders which in typical wind speed ranges shed vortices that can drive strong HGM sway, and the extended length with coexisting antenna is particularly problematic. Hardly any full-scale experimental data exist for HGMs leading to considerable uncertainty and conservatism in dynamic structural assessments required before installing new antenna. Such an assessment of the 235m HGM at Moel-y-Parc (MYP) in North Wales by COWI required installation of vibration mitigation measures, in the form of a pair of tuned liquid dampers, to mitigate risk of adverse dynamic performance similar to the design for earthquakes (Banerji et al., 1992). To check operation of the dampers and to provide 
additional information on dynamic characteristics a structural health monitoring system was installed at the same time as the dampers. In addition, the data recorded during the monitoring will help to study the full-scale behaviour of those structures in order to advance the very limited understanding of HGM dynamic parameters, especially damping. Also, according to Sparling \& Wegner (2006) the non-linearities introduced by the varying sag in the stay cable used for horizontal support and the large displacements by the mast itself modify natural frequencies and mode shapes. As part of the MYP study, the simplified static analysis assumptions developed by Davenport and Sparling (1992) will be verified and updated.

\section{Moel-y-Parc high guyed mast}

Moel-Y-Parc station was built in 1962 with the requirement to achieve at least $750 \mathrm{ft}(229 \mathrm{~m})$ to enable UHF coverage of coastal resorts from Conway to Prestatyn, given the special orography near to the shore. The radiation pattern was approximately semi-circular, $25 \mathrm{~kW}$ being radiated from south-east to northwest with reduced power north and east so as to cover the Welsh areas but avoiding overlap with English areas already served. In addition, it provides supplementary service to Cheshire, Manchester and Lancaster counties, areas with strong importance due to high population.

Moel-Y-Parc HGM, the large violet triangle Southern-West from Liverpool in Figure 1(a), is the main station of the area coloured in dark blue, sharing with other adjacent stations (small triangles) to amplify the coverage in field. In light blue, the mentioned supplementary coverage area is indicated.

Apart from the UHF equipment focused on Television Broadcasting, MYP holds antennas from several customers with a range of frequencies. Presently, the VHF Digital Radio antenna is installed at $188 \mathrm{~m}$, and below $150 \mathrm{~m}$, with other capacity taken by the telecoms sector, with customers such as EE, Airwave Solutions, 3TM and CTIL using a variety of equipment like sector panels, dishes or dipoles, as well as the corresponding cabling and feeders for each antenna running internally.

The structure of the mast comprises two different sections of triangular lattice towers, with four stayed levels. The $1 \mathrm{st}$ section between ground and the 3rd stay level at $171.7 \mathrm{~m}$ is $2.6 \mathrm{~m}$ wide, and the last stretch until the 4 th stay level decreases to $1.9 \mathrm{~m}$. Finally, the total height of $240 \mathrm{~m}$ is achieved with a cantilever spine antenna of $12 \mathrm{~m}$ based on a lattice tower of $1 \mathrm{~m}$ width.

The mast is designed to be stiffer at ground levels with larger leg sizes and more complex steelwork configuration to avoid buckling failure. Legs at ground lowest levels are solid round rods of $146 \mathrm{~mm}$ unlike the 4 th level that uses $95.2 \mathrm{~mm}$ rods, and equal angles are used for bracing and redundancy. All members are made of mild steel, although further strengthening has used steel with better quality. Additionally, the connections are mainly bolted while there are few welded joints on crowded nodes.
Figure 1 Moel-Y-Parc HGM

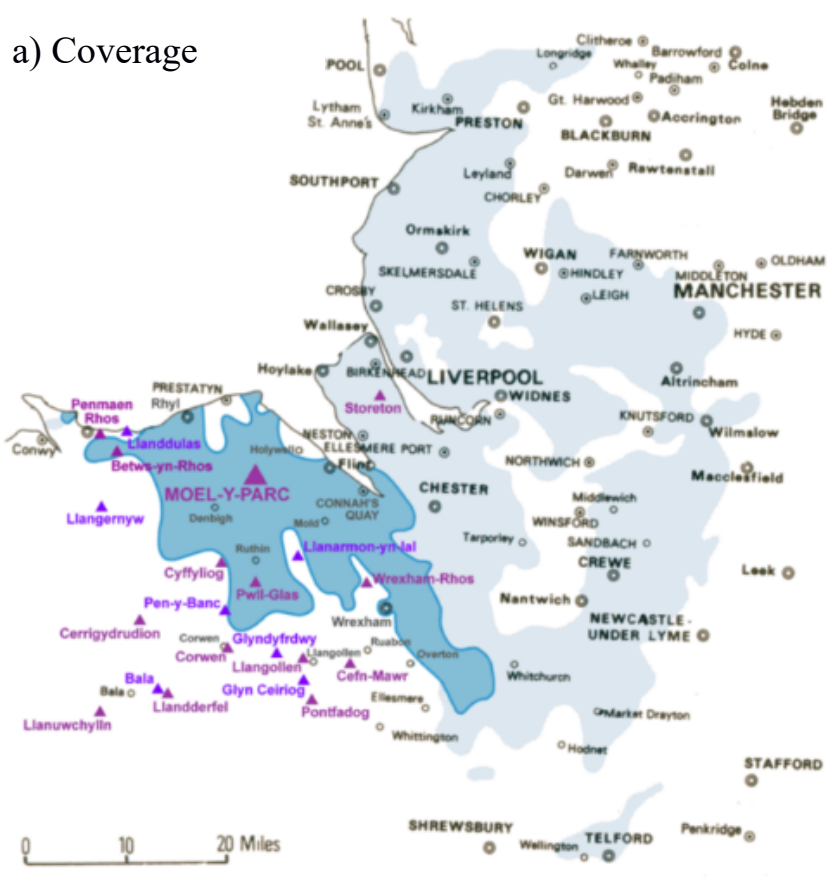

b) Elevation

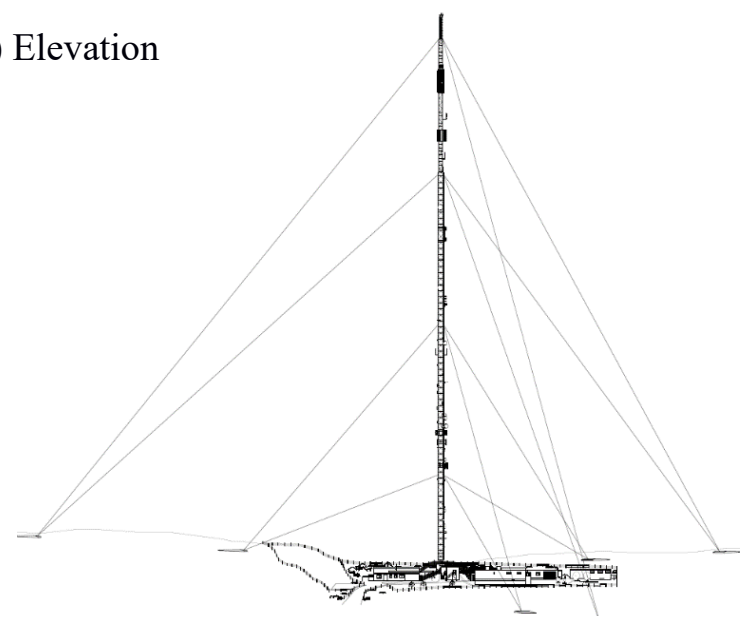

c) Site plan
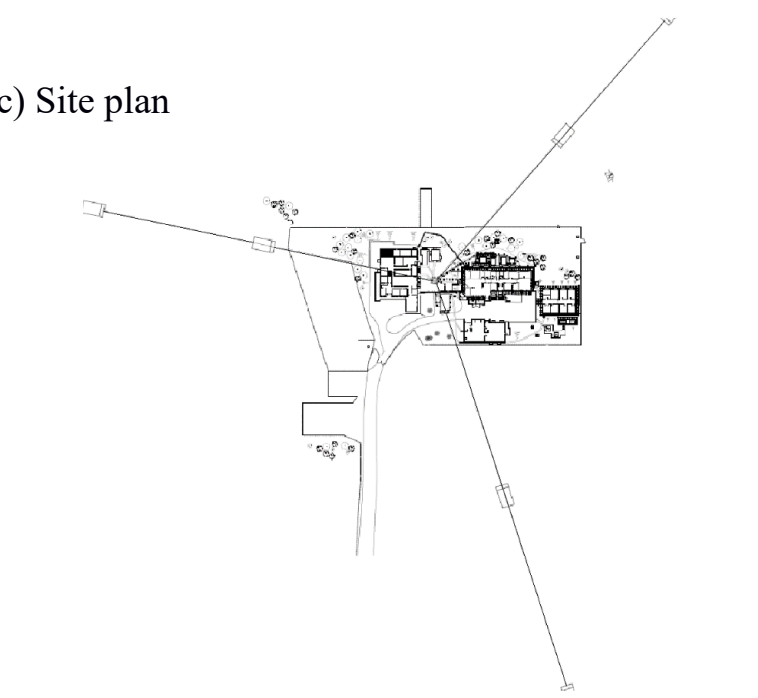
The mast is held at four stay levels with three stay lanes at each level (12 stays in total) with orientation $92^{\circ}, 212^{\circ}$ and $332^{\circ}$. Due to the orography, each stay has its own configuration of diameter, initial tension, protection or geometry which are defined to provide enough tension at each joint level. Finally, the stays are attached to concrete foundation blocks in pairs as the figure.

The standard loading design is defined by the buffeting gust wind speed coming from south-west which is heavily amplified by the orography of the location. Also, due to the characteristics of the antenna, an aeroelastic effect around the mast shall induce large movements which could risk the structural integrity of the mast. Expertise consulting approaches show that vortex shedding could excite modes of vibration around $0.62 \mathrm{~Hz}$ and the "lock in" effect under this vortex shedding issue could induce a peak horizontal acceleration of $0.9 \mathrm{~g}$ at antenna levels. The dynamic effect added to the existing static loading and possibility of fatigue failure would present a significant risk to the safety of any person around the antennas.

To mitigate those vortex-shedding vibrations, a tuned liquid damper was designed and installed, aiming to achieve $10 \%$ damping, which could assure a good behaviour of the mast.

\section{Structural health monitoring system}

A structural health monitoring (SHM) system was designed to record mast vibrations to assure its structural integrity, verifying that the mast behaves as expected, the TLD helps to decrease possible movements and there are no risks under several weather conditions. For that, the system is based on 5 biaxial acceleration measurement boxes placed at key levels as shown in Table 1.

In addition, in order to correlate different amplitudes of the response with wind conditions, an anemometer was added to the system. Originally designed to be placed at the top of the mast, due to complications during initial installation, it was finally moved to a platform level at $225 \mathrm{~m}$, just below the 4 th stay level, as indicated in Figure 2.

Designing and implementing instrumentation on the mast was found to be extremely challenging due to a possibility of radio frequency interference (RFI) on the signal cables of a conventional centralised DAQ system, due to the high RF energy from the antennas. In order to minimise such RFI, the sensor output voltage needs to be acquired at the sensor location suggesting a distributed DAQ system. However, such distributed DAQ brought introduced a synchronisation problem between the six sensor nodes. To address this, the GPS-based time-synchronisation method for wireless sensors developed by the author (Koo et al., 2019) was used. In each accelerometer or anemometer box, measured data are accurately time-stamped by the time information provided by a GPS module and then resampled for time-synchronised measurements. For immunity of RFI on the data-transfer from the DAQ boxes to the base station on the ground, a fibre-optic media converter was used in each DAQ box for establishing an Ethernet connection to the base station. The base station was connected to Internet connection via a $4 \mathrm{G}$ mobile router. A
$110 \mathrm{~V}$ main power cable was installed along the length of the mast to provide a permanent power to the six boxes.

Figure 2 Installed position of Accelerometer box (ACC5) and Anemometer

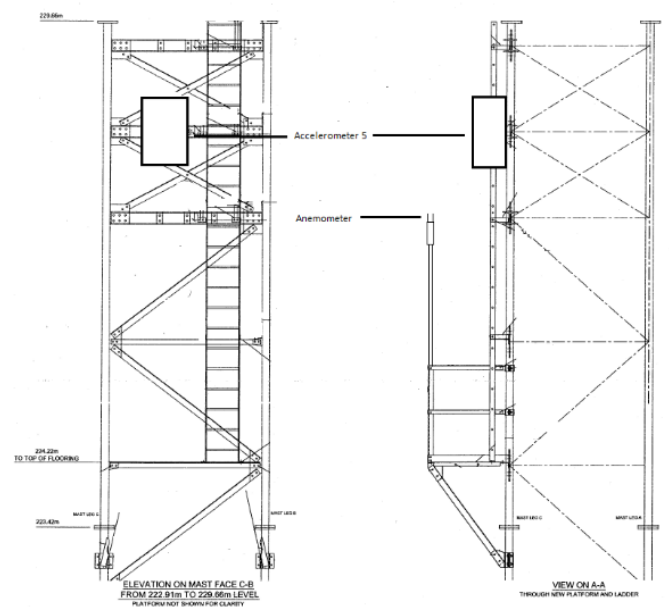

Colibrys VS1002A MEMS accelerometer are used two per box for biaxial horizontal acceleration measurement. VS1002A is a good-performance accelerometer of noise floor $=7 \mathrm{ug} / \mathrm{sq}(\mathrm{Hz})$ with a moderate cost of $£ 180$ as of 2018 . The anemometer is a Young 85004 ultrasonic anemometer, having a RX-232 interface for measured output. Figure 3 shows the sensors.

\section{Table 1 SHM component locations}

\begin{tabular}{lll}
\hline Sensor & Abbr. & Position \\
\hline Anemometer & WND1 & Platform level, 225m \\
$5^{\text {th }}$ Acc'rometer & ACC5 & Forth stay level, 230m \\
$4^{\text {th }}$ Acc'rometer & ACC4 & TLD level, 212m \\
$3^{\text {th }}$ Acc'rometer & ACC3 & Third stay level, 172m \\
$2^{\text {rd }}$ Acc'rometer & ACC2 & Mid-span of second section, \\
& & $140 \mathrm{~m}$ \\
$1^{\text {st }}$ Acc'rometer & ACC1 & Second Stay Level, 108m \\
\hline
\end{tabular}

Figure 3 Young 85004 ultrasonic anemometer (left) and Colibrys VS1002A MEMS accelerometer (right)
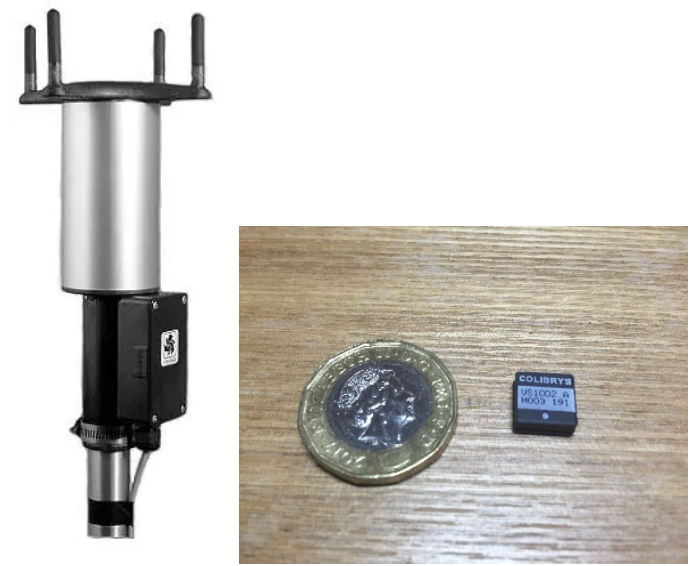
Sensor node in the acceleration DAQ boxes were built using a Raspberry Pi (https://www.raspberrypi.org) stacked with 1) an Arduino Mega2560 compatible custom-made board (GOM board) having GPS-based time-stamping capability, 2) an Analogue-to-Digital conversion custom-made board (ADC board) controlled by the GOM board, and 3) a 2.8" touchscreen TFT display (https://www.adafruit.com/product/1601) as shown in Figure 3 (right). Two VS1002A sensors placed in the DAQ box perpendicular to each other (see Figure 3 (middle)) were connected to the ADC board using a 24-bit 4 channel simultaneous ADC Texas Instrument ADS1274 chip. The GOM board has a GPS module FGPMMOPA6H (GlobalTop Tech, 2012) feeding the accurate time information to the Arduino Mega2560 MCU. The acquired data and corresponding timestamps were sent to UART RX pin of the Raspberry Pi 3 which has a python script performing the resampling technique (Nagayama \& Spencer, 2007) with resampled data stored in the Raspberry Pi SD memory card. Adafruit PiTFT screen was used as a console monitor of the Raspberry Pi 3 to show status of DAQ operation with optional real-time graphs of time-history or power spectral density. The Raspberry Pi 3 ethernet ports are connected to Fibre-option Media Converter for data transfer from the SD memory card to the HDD of the base station. The base station has a set of python scripts to upload the data files into a secure ftp server at Exeter executed every two hours.

The sensor node in anemometer DAQ box was built using a Raspberry Pi and a custom-made GPS module board directly feeding the time-information to the Raspberry Pi via gpsd (http://catb.org/gpsd). A python script on the Raspberry Pi was used to parse UART outputs from the anemometer and to store data into the SD memory card. Figure 4 shows the SHM hardware assembled.

\section{Data interpretation}

Evaluation of the time-varying response data is performed for specific responses such as gust-induced buffeting identified by high wind speed, or vortex shedding at low winds for a specific vibration mode. Among other purposes, the system shall verify the structural integrity of the structure in real time, advising strange movements, and tracking frequencies and damping values.

To that end, the SHM system creates daily and weekly reports with the data obtained during the records, aiming to improve condition monitoring and to provide data for HGM performance research. Those reports include plots of the time history and PSD of all accelerometers as Figure 5, with the corresponding orbit plot and PSD for the damper level shown in Figure 6.
Figure 4 SHM HW System: (top) testing of the boxes, (middle) ACC box, and (bottom) GOM node
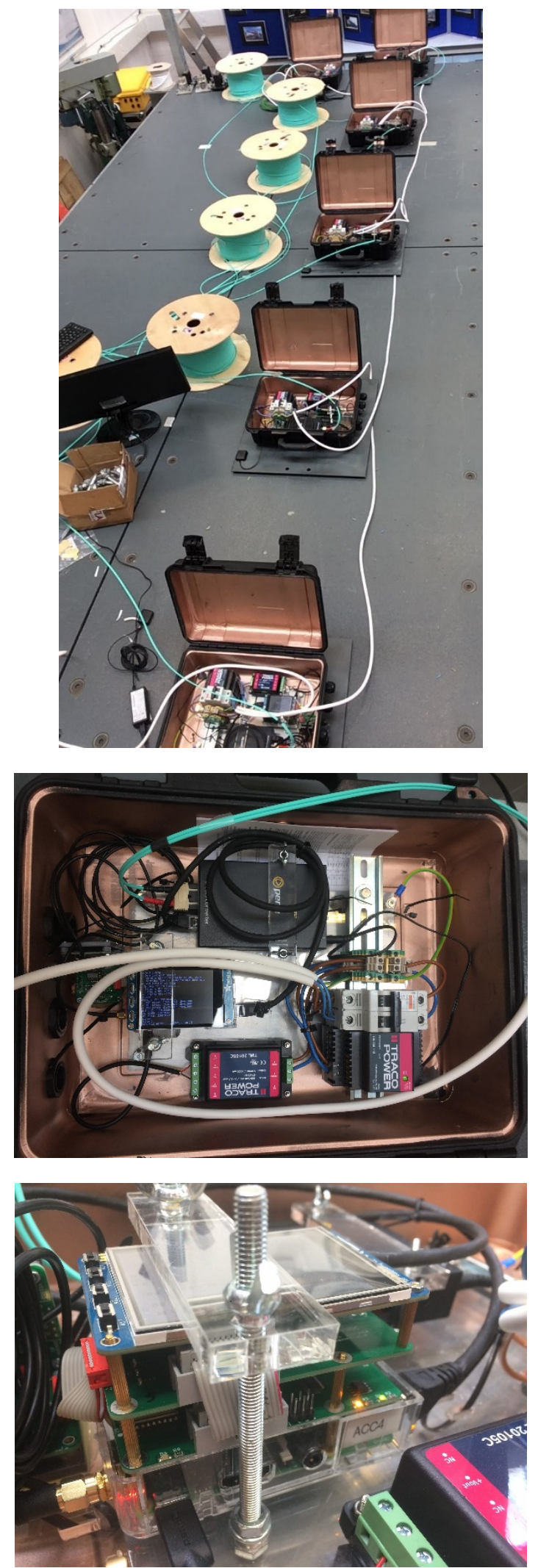
Figure 5 Acceleration time history and PSD
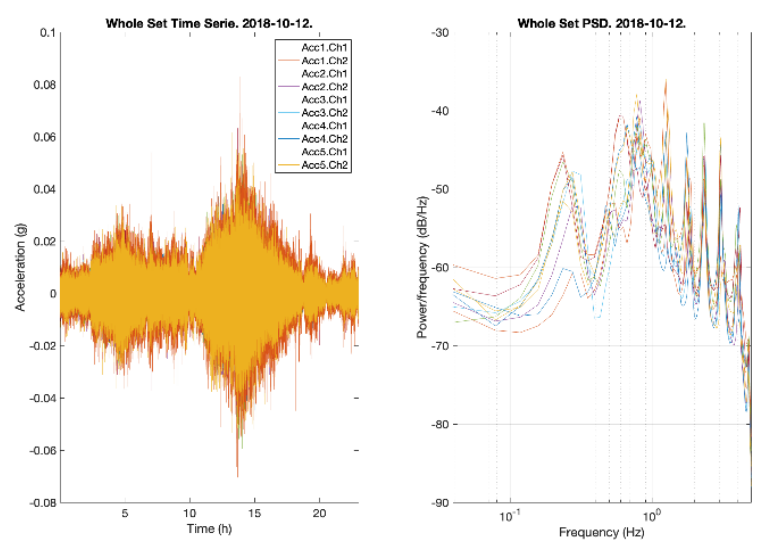

Figure 6 Orbit plot and PSD of acceleration at the TLD location.
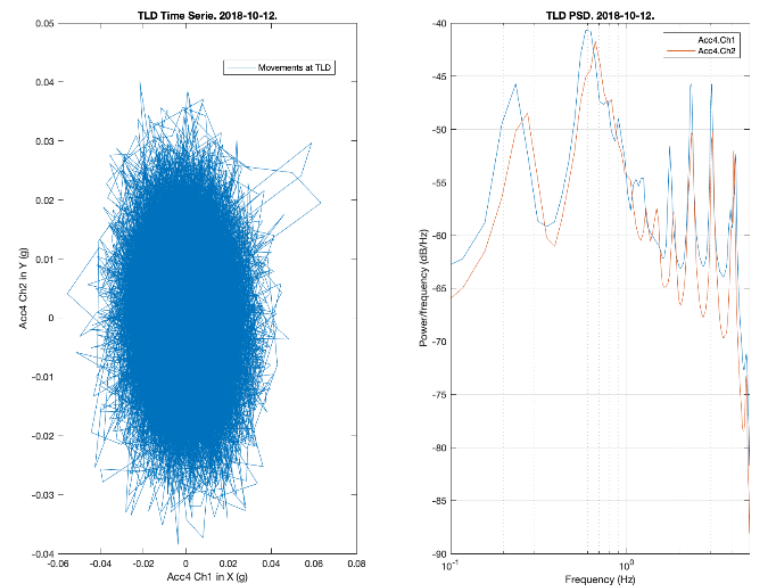

The selected time frame belongs to a strong event on October $12^{\text {th }}, 2018$ in which there is no sign of vortex shedding (this would be a strong alignment of response in a single direction with obvious narrow elliptical orbit plot. The consultant assessments on this specific mast pre-advised a critical peak horizontal acceleration at antenna levels around $0.8 \mathrm{~g}$. The response does not achieve high values on this case which suggests that the damper works correctly, and the structure performs within capacity.

On the other hand, the Figures 5 and 6 show the frequency content of the response due to wind loading. The high modal density corresponds to a complicated performance of a high complexity structure, with as many as six vibration modes in the range 0.2 to $0.3 \mathrm{~Hz}$. This known behaviour complicates a lot any kind of static approach analysis.

To compare response and loading, the ultrasonic anemometers working at $4 \mathrm{~Hz}$ sample frequency provide wind speed and wind direction, Figure 7.
Figure 7 Wind speed and direction time history (top) and wind-rose plot (bottom)
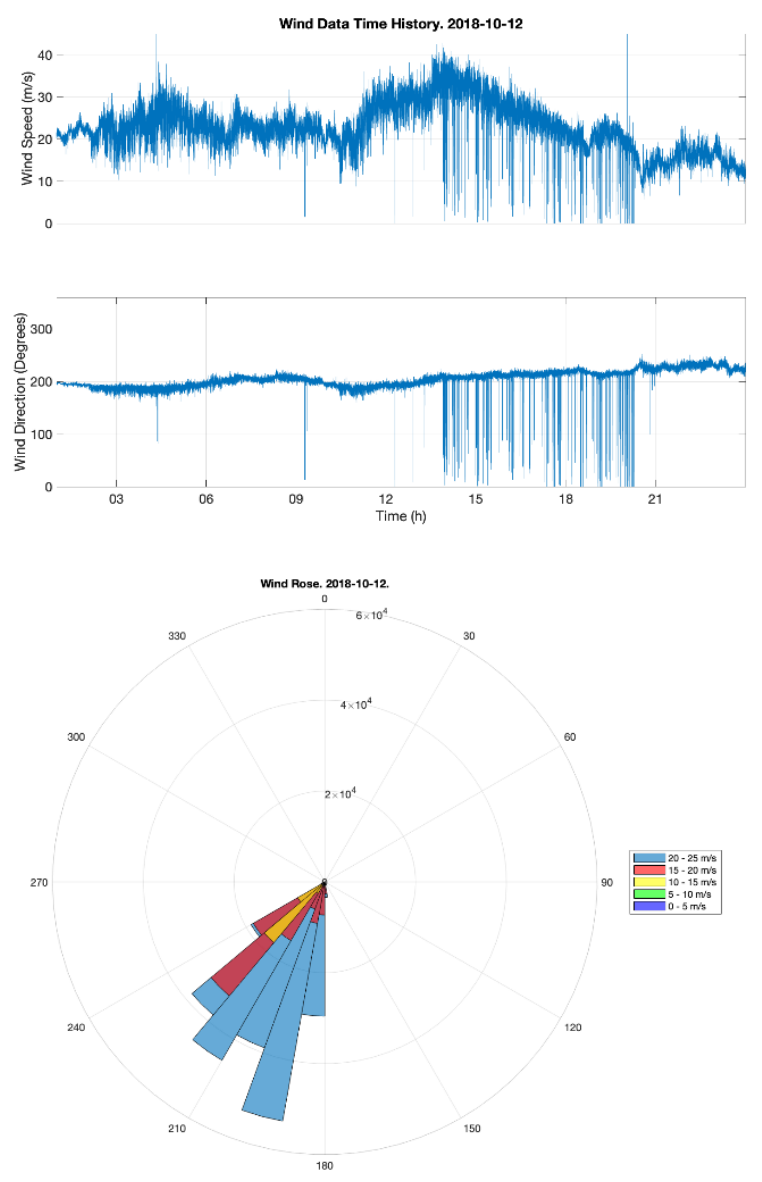

The monitoring reports also provide important information for each hour, e.g. RMS and maximum and minimum values, advising which time frames to select for a manual and focused analysis.

\section{Summary and conclusion}

This paper presented the innovative sensing solution developed and deployed for structural health monitoring of the Moel-YParc High Guyed Mast, in North Wales. The conventional analogue voltage signal cables were avoided due to a high possibility of radio frequency interference by the high radiofrequency density from the antennas on the long cables along the height of the structure of $240 \mathrm{~m}$. The innovative distributedDAQ sensing system has been developed and deployed based on the new technology of Arduino, Raspberry Pi and GPSbased time-synchronisation as described below.

- The Arduino Mega 2560 compatible board with a low-cost GPS module has been developed to timestamp measured data very accurately and precisely with the maximum time-stamping error of 1 micro-second.

- $\quad$ The 24-bit 4 channel simultaneous ADC board using Texas Instrument ADS1274 ADC chip, controlled by the Arduino board, has been developed to measure acceleration signals from the good-performance, 
moderate cost MEMS accelerometer of Colibrys VS1002A.

- Raspberry Pi fed by the time-stamped data by the Arduino compatible board, performed the resampling technique to generate time-synchronised data based on accurately time-stamped data.

- Fibre-optic Ethernet network was used for data communication between the sensing nodes and the base station to work reliably under the radiofrequency interference. The $4 \mathrm{G}$ router attached to the base station network provided Internet access to upload measured data files into the server at Exeter.

From the on-going work of data-interpretation of the measured vibrations, followings conclusions can already be drawn.

- Under $40 \mathrm{~m} / \mathrm{s}$ wind-speed at $12^{\text {th }}$ Nov 2018, the maximum acceleration response measured was $0.08 \mathrm{~g}$, which is far less than the critical response $0.8 \mathrm{~g}$ indicating a successful operation of the tuned liquid damper.

\section{References}

Banerji P, Murudi M, Shah A and Popplewell N (1999) Tuned Liquid Dampers for Control of Earthquake Response. 13th World Conference on Earthquake Engineering13th World Conference on Earthquake Engineering, 587-602 August

Davenport AG and Sparling BF (1992) Dynamic gust response factors for guyed towers. Journal of Wind Engineering and Industrial Aerodynamics, 2237-22484311992.

GlobalTop Technology Inc (2012) FGPMMOPA6H GPS Standalone Module Datasheet.

Koo KY et al. (2019) Time Synchronisation for Wireless Sensors Using Low-Cost GPS Module and Arduino, Frontiers in Built Environment, doi: 10.3389/fbuil.2018.00082

Nagayama T, and Spencer BF (2007) Structural Health Monitoring Using Smart Sensors, Report No. NSEL-001. Urbana-Champaign.

Sparling BF, Wegner LD (2006) Comparison of frequencyand time-domain analyses for guyed masts in turbulent winds. Canadian Journal of Civil Engineering, 169-182332

WG4 IASS (1981) Recommendations for guyed masts, International Association for Shell and Spatial Structures 